\title{
MODULATION OF GPCR PHARMACOLOGY FROM THE EXTRACELLULAR VESTIBULE
}

\section{G.M. Keserü}

Medicinal Chemistry Research Group, Research Center for Natural Sciences, Hungary

DOI: 10.19163/MedChemRussia2021-2021-56

E-mail: keseru.gyorgy@ttk.hu

G-protein coupled receptors (GPCRs) are considered important therapeutic targets due to their pathophysiological significance and pharmacological relevance. Endogenous ligands bind to the orthosteric binding pocket (OBP) embedded in the intrahelical space of the receptor. During the last years, however, it has been turned out that in many receptors there is secondary binding pocket (SBP) located in the extracellular vestibule that is much less conserved. In some cases it serves as a stable allosteric site harbouring allosteric ligands that modulate the pharmacology of orthosteric binders. In other cases it is used by bitopic compounds occupying both the OBP and SBP. In these terms, SBP binding moieties might influence the pharmacology of the bitopic ligands. Together with others, our research group showed that SBP binders contribute significantly to the affinity, selectivity, functional activity, functional selectivity and binding kinetics of bitopic ligands.

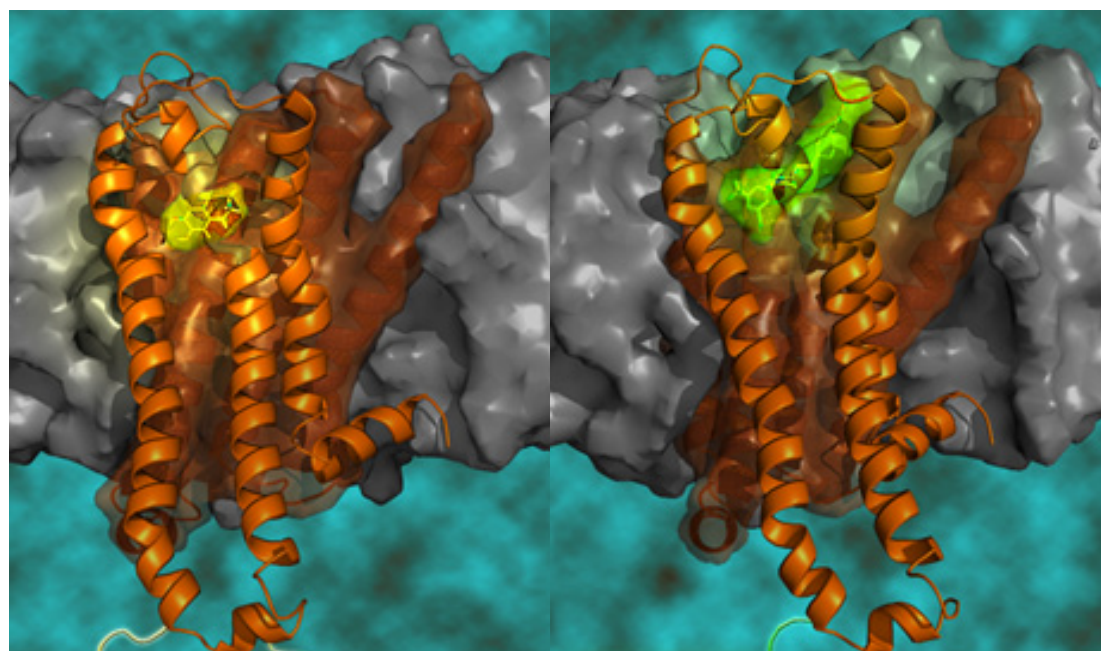

\section{References}

[1] G. M. Keserű et al. ACS Med. Chem. Lett. 2014, 5, 1010-1014

[2] G. M. Keserű et al. Chem. Commun., 2020,56, 14167-14170

[3] G. M. Keserü et al. Bioorg. Chem.2021, 111, 104832. 